\title{
Leitthema
}

Hautarzt 2014 • 65:312-320

DOI 10.1007/s00105-013-2705-z

Online publiziert: 11. April 2014

๑) Springer-Verlag Berlin Heidelberg 2014

E. Haneke 1, 2, 3, 4

${ }^{1}$ Dermaticum Freiburg

2 Dermatol Klinik Inselspital, Univ. Bern

${ }^{3}$ Centro Dermatol Epidermis, Porto

${ }^{4}$ Kliniek voor Huidziekten, Academisch Ziekenhuis, Univ. Gent

\section{Maligne Tumoren des Nagelorgans}

„Warze“ vorstellt. Auch dann vergeht oft noch viel Zeit, bis eine Biopsie und schließlich die Exzision durchgeführt werden. Mit molekularbiologischen Methoden wurden in den letzten 20 Jahren Hochrisikopapillomviren in vielen $\mathrm{Tu}$ moren nachgewiesen, besonders HPV 16, aber auch 18, 34, 35, 73 und viele weitere $[11,12]$. Multiple HPV-Typen im selben Tumor kommen vor $[13,14]$. Wenn Hochrisiko-HPV nachgewiesen werden können, sind meist die Finger 2-4 betroffen, die Patienten sind etwas jünger, und Frauen überwiegen $[15,16]$. Eine (ano)genitodigitale Übertragung wurde schon lange angenommen [17, 18, 19, 20]. Chronische Röntgenstrahlen- und Arsenexposition, lang dauernde Infektion, Trauma [21] und Narben sind weitere mögliche Ursachen.

\section{> Die Progression des Morbus Bowen zum Bowen-Karzinom ist eher selten.}

Sie kündigt sich durch Knotenbildung an. Metastasierung ist eine Ausnahme. Weil man nicht sicher sein kann, ob wirklich nur in den untersuchten Schnittebenen ein Carcinoma in situ besteht, wurde für Morbus Bowen und das Plattenepithelkarzinom des Nagels die zusammenfassende Bezeichnung epidermoides Karzinom vorgeschlagen [22].

Ein Plattenepithelkarzinom des „klassischen Typs" kann auch direkt entstehen. Die Klinik ist gewöhnlich noch weniger charakteristisch. Die meisten Plattenepithelkarzinome entstehen unter dem Daumennagel und führen zu einer schmerzlosen Onycholyse mit Nässen (• Abb. 2). Der Nagel hebt sich ab und wird gelb, oft auch grünlich durch sekundäre Pseudomonas-aeruginosa-Kolonisation. Schmerzen können als Zeichen einer Knochenerosion angesehen werden. Fortgeschrittene Tumoren imponieren als blutende exophytische Gebilde, die mehr als nur das Nagelbett betreffen [23]. Das Wachstum ist langsam infiltrativ. Eine Knochenerosion ist selten [24].

Die Therapie der Wahl des ungualen Morbus Bowen und Plattenepithelkarzinoms ist die mikroskopisch kontrollierte Chirurgie ( Abb. 1, 2, [25]), beim Insitu-Karzinom eventuell in Kombination mit Tazaroten, 5-Fluoruracil und Imiquimod [26]. Imiquimod und photodynamische Therapie wurden ebenfalls angewandt [27], sind nach unserer Erfahrung aber sehr oft nicht ausreichend wirksam. Eine Amputation ist bei Knochenbeteiligung erforderlich. Die Radiotherapie wurde als Alternative bei nicht resezierbarem subungualem Karzinom beschrieben [28], jedoch muss diese Aussage

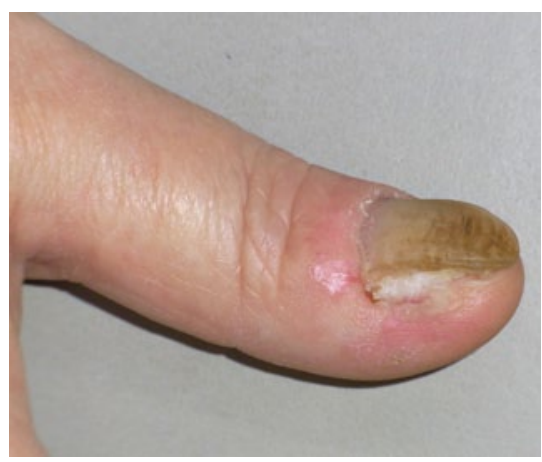

Abb. $1 \Delta$ Ausgedehnter Morbus Bowen des Nagels mit Einbeziehung von Nagelbett, distaler Matrix, proximalen und distalen Nagelwällen 
Hier steht eine Anzeige.

黛 Springer 


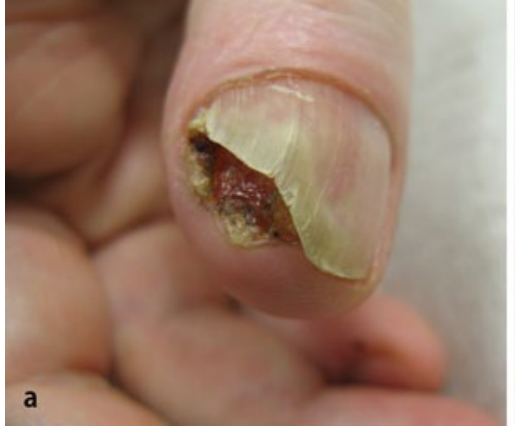

Abb. $2 \Delta$ Plattenepithelkarzinom des Nagelbetts des Daumens. a Vor Therapie. b 4 Monate nach der Operation

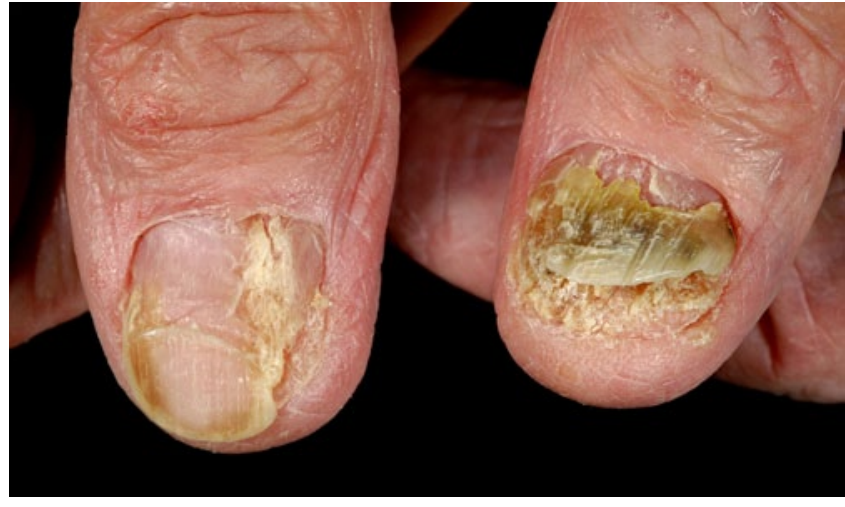

angezweifelt werden, da eine Fingeramputation immer möglich ist.

Die Differenzialdiagnose umfasst vulgäre Warzen, Cornu cutaneum [29], Keratoakanthom, chronisches Ekzem, Paronychie, chronische Ulzeration, Granuloma teleangiectaticum, Melanom, Metastasen und andere Tumoren. Sie ist besonders schwierig, wenn ein Patient 2 verschiedene Veränderungen, z. B. Morbus Bowen und Melanoma in situ, hat [30]. „Jede Warze am Nagel bei Patienten mit Genitalwarzen, Dysplasie oder Immunsuppression sollte als potentielles Karzinom angesehen werden." [31] Entfernung des den Krankheitsprozess bedeckenden $\mathrm{Na}$ gels ist essenziell für eine klinische Diagnose.

\section{Andere Plattenepithelkarzinome}

Verruköses Karzinom und Carcinoma cuniculatum sind niedrig maligne, langsam wachsende Plattenepithelkarzinome. Sie sind selten und weisen keine pathognomonischen klinischen Zeichen auf [32, 33, 34]. Meist ist die Groß- oder Kleinzehe betroffen, Finger nur ausnahmsweise [31]. Eine Invasion an bzw. in den Kno-

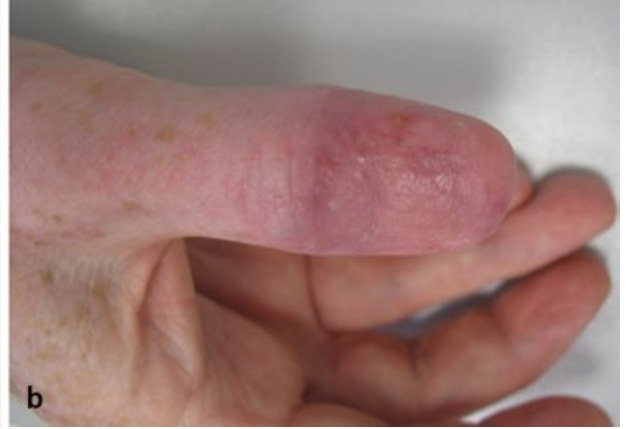

Abb. $3<$ Spezifische Nagelveränderungen beim Sézary-Syndrom

\section{Aggressives digitales papilläres Adenokarzinom}

Dieses Karzinom wurde ursprünglich als aggressives digitales papilläres Adenom bezeichnet. Da es rezidiviert und metastasiert, wird es jetzt als maligner Schweißdrüsentumor angesehen $[41,42]$. Es kommt praktisch ausschließlich an Fingern, Zehen, Handflächen und Fußsohlen vor mit Bevorzugung der Hand. Die Patienten sind meist zwischen 40 und 60 Jahre alt, aber auch jüngere $\mathrm{Pa}$ tienten wurden beobachtet $[43,44,45]$. Der Tumor wächst langsam und unauffällig, ist gummiartig fest, grau bis rosaweiß, manchmal zystisch und bildet einen Knoten oder eine Infiltration, besonders zwischen Nagelbett und distalem interphalangealem Gelenk. Eine Ulzeration ist häufig. Obwohl er mehrere Zentimeter groß werden kann, wird die Beweglichkeit des Gelenks nicht eingeschränkt. Schmerzen sind Zeichen einer Invasion von Knochen, Gelenk oder Nerven.

\section{$>$}

Etwa die Hälfte dieser Karzinome rezidiviert nach primärer Exzision, und $40 \%$ metastasieren, meist in die Lunge.

\section{Basalzellkarzinom}

Basalzellkarzinome (BCC) sind entsprechend ihrer postulierten Herkunft vom Haarfollikel [36] am Nagelorgan sehr selten; nur etwa 25 wurden bisher beschrieben. Sie erscheinen meist als chronische Paronychie mit Ulzeration oder erosiven Tumoren des proximalen Nagelwalls, die an ein Granuloma teleangiectaticum oder Granulationsgewebe erinnern [37]. Ein BCC war pigmentiert [38]. Die Anamnese betrug zwischen 1 und 40 Jahren [39]. Die Therapie der Wahl ist die komplette chirurgische Entfernung [40].

\section{Andere Karzinome}

Nagelspezifische Karzinome sind das onycholemmale Karzinom und der maligne onycholemmale Tumor. Ihre Differenzierung ist nicht eindeutig. Beide ähneln histologisch einem malignen proliferierenden Trichilemmtumor.
Radikale Exzision oder Amputation gelten als Therapie der Wahl [46]. Differenzialdiagnostisch muss an Zysten und Pseudozysten, Granuloma teleangiectaticum, andere Angiome, Fremdkörpergranulom, Gicht, Plattenepithelkarzinom, Epitheloidzellsarkom, Riesenzelltumor, Osteitis terminalis und andere Weichteilinfektionen gedacht werden.

\section{Ekkrines Porokarzinom}

Das ekkrine Porokarzinom ist selten und wird vorzugsweise bei älteren Menschen an Handtellern und Fußsohlen beobachtet $[47,48]$. Es tritt nur ausnahmsweise an der Fingerspitze auf [49], aber ein Porokarzinom der Umgebung kann auch das Nagelorgan komplett überwachsen. Chronische Röntgenstrahlenexposition scheint eine mögliche Ursache zu sein [50]. Vorkommen im lateralen Nagelfalz simulierte ein Plattenepithelkarzinom [51]. Möglicherweise entsteht es auch durch maligne 
Transformation eines präexistenten ekkrinen Poroms [52].

\section{Adenozystisches Schweißdrüsenkarzinom}

Die muzinöse Variante des seltenen adenozystischen Schweißdrüsenkarzinoms wurde an der Spitze der Großzehe einer 30-jährigen Frau beobachtet. Der Tumor war beweglich, aber schmerzempfindlich [53].

Die Beziehungen bzw. die Unterscheidung von adenoid-zystischem und mikrozystischem Adnexkarzinom sind nicht eindeutig geklärt.

\section{Spiradenokarzinom}

Dieses im Allgemeinen aus einem benignen Spiradenom entstehende Karzinom ist sehr selten. Anamnestisch können multiple ekkrine Spiradenome vorliegen [54]. Bisher wurde ein Fall eines Spiradenokarzinoms des Nagelbetts beschrieben, das aus einem Spiradenom der dorsolateralen Großzehe nach multiplen Kauterisationen und einer lokalen Exzision hervorgegangen war [55].

\section{Hidradenokarzinom}

Dieser seltene Tumor ist unter verschiedenen Bezeichnungen wie papilläres Klarzellkarzinom, Klarzellhidradenokarzinom, malignes Klarzellhidradenom, malignes Klarzellakrospirom, malignes ekkrines Akrospirom, noduläres Hidradenokarzinom, mukoepidermoides Hidradenokarzinom und malignes noduläres Klarzellhidradenom beschrieben worden. Es ist ein langsam wachsender, solitärer Tumor, der keine Beschwerden verursacht. Zwei solcher Karzinome wurden im Nagelapparat beschrieben, einer im Mittelfingernagelbett, der als längs verlaufender Pigmentstreifen begann, sich schnell vergrößerte und bei der Konsultation einen halbkugeligen ulzerierten Tumor darstellte, der vom Nagelbett bis zum proximalen Nagelbett reichte [56]. Ein anderes Hidradenokarzinom imponierte als Onychomykose.

Hautarzt 2014 • 65:312-320 DOI 10.1007/s00105-013-2705-z

(c) Springer-Verlag Berlin Heidelberg 2014

\section{E. Haneke}

\section{Maligne Tumoren des Nagelorgans}

\section{Zusammenfassung}

Entsprechend den unterschiedlichen Geweben, die das Endglied von Fingern und Zehen aufbauen, gibt es sehr viele verschiedene Tumoren. Am häufigsten ist der Morbus Bowen, der meist als verruköse Veränderung bei Personen ab dem Alter von 40 Jahren auftritt. Hauptlokalisationen sind der laterale Nagelwall und das Nagelbett. Nach jahrebis jahrzehntelangem Bestand ist der Übergang in ein invasives Plattenepithelkarzinom möglich. Dieses kann sich aber auch primär subungual entwickeln, meist als Onycholyse mit Nässen. Der zweithäufigste maligne Nageltumor ist das Melanom. Wenn es von der Matrix ausgeht, ist es meist pigmentiert, während Nagelbettmelanome überwiegend amelanotisch sind und oft als Unguis incarnatus bei älteren Personen imponieren. Therapie der Wahl ist bei in situ und früh invasiven

\section{Malignant nail tumors}

\begin{abstract}
Because of the large number of different tissues making up the distal phalanx of fingers and toes, a large variety of malignant tumors can be found in and around the nail apparatus. Bowen disease is probably the most frequent nail malignancy. It is usually seen as a verrucous plaque of the nail fold and nail bed in persons above the age of 40 years. It slowly grows over a period of years or even decades before degenerating to an invasive squamous cell carcinoma. The latter may also occur primarily often as a weeping onycholysis. The next most frequent nail malignancy is ungual melanoma. Those arising from the matrix are usually pigmented and often start with a longitudinal melanonychia whereas those originating from the nail bed remain amelanotic, are often nodular and mistaken for an ingrown nail in an elderly person. The
\end{abstract}

Melanomen die großzügige Lokalexzision unter Erhalt der Endphalanx. Amputation ist nur bei fortgeschrittenen Melanomen indiziert. Neben den beiden häufigen ungualen Malignomen gibt es selten nagelspezifische Karzinome, maligne Gefäß- und Knochentumoren sowie andere Sarkome, Beteiligung im Rahmen von malignen Systemerkrankungen und Metastasen. Sie lassen sich in der Mehrzahl der Fälle nicht klinisch eindeutig diagnostizieren. Trotzdem muss an einen malignen Tumor gedacht werden bei allen umschriebenen, auf konservative Therapie nicht ansprechenden Nagelprozessen.

Schlüsselwörter

Nägel · Morbus Bowen · Karzinom ·

Melanom · Sarkom

\section{Talgdrüsenkarzinom}

Dieses Karzinom tritt zu mehr als 50\% an den Augenlidern auf, und nur 1 Fall wurde an der Fingerlateralseite eines 46-jährigen Mannes beschrieben. Es handelte sich um eine schmerzlose Schwellung, die unter dem Verdacht einer Zyste exzidiert wurde [57]. treatment of choice for in situ and early invasive subungual melanomas is generous extirpation of the nail apparatus whereas distal amputation is only indicated for advanced melanomas. In addition to these frequent nail malignancies, nail-specific carcinomas, malignant vascular and osseous tumors, other sarcomas, nail involvement in malignant systemic disorders and metastases may occur. In most cases, they cannot be diagnosed accurately on clinical grounds. Therefore, a high degree of suspicion is necessary in all isolated or single-digit proliferations that do not respond to conservative treatment.

Keywords

Nails - Bowen's disease - Carcinoma .

Melanoma $\cdot$ Sarcoma

\section{Sarkome}

Am Nagel wurden einzelne Fälle verschiedener Sarkome meist als Einzelbeobachtungen beschrieben.

> Von allen Sarkomen ist das Dermatofibrosarcoma protuberans am häufigsten. 


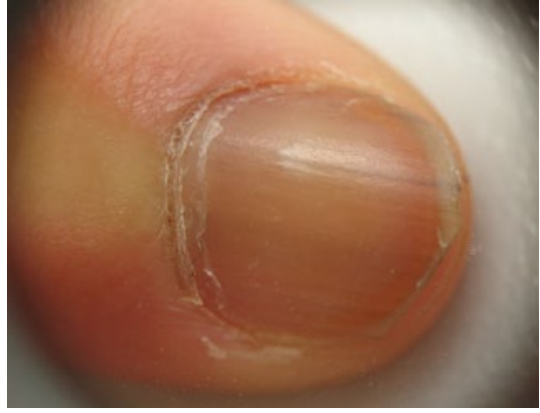

Abb. $4 \Delta$ Melanoma in situ bei einem 11-jährigen Mädchen. Seit dem Alter von 18 Monaten hatte ein schmaler brauner Streifen im Nagel bestanden, der sich knapp 2 Jahre vor der Erstvorstellung plötzlich verbreiterte und unregelmäßig wurde

Nur 3 Dermatofibrosarcoma protuberans (DFSP) wurden am Nagelorgan beschrieben: ein rosafarbener gelappter Tumor am Daumenendglied einer 31-jährigen Frau [58], ein dunkles hyperkeratotisches Infiltrat am proximalen Nagelwall der Großzehe eines 53-jährigen Mannes [59] und ein subunguales DFSP [60]. Die klinischen Differenzialdiagnosen waren Warze, Keloid, hypertrophische Narbe, rezidivierende digitale Fibromatose und chronische Paronychie. Die histologische Differenzialdiagnose umfasst sowohl benigne als auch maligne fibrozytäre Prozesse.

Das Epitheloidzellsarkom ist ein langsam in der Nachbarschaft von Gelenken wachsender Tumor bei jungen Menschen [61]. Meist wird die Diagnose erst gestellt, wenn das Sarkom schon ulzeriert und metastasiert ist. Einblutung kann zu dunkler Pigmentierung führen. An der Endphalanx kann eine myxoide Pseudozyste imitiert werden. Trotz Amputation kommt die Therapie oft zu spät [62]. Differenzialdiagnostisch sind zahlreiche andere zu Ulzeration neigende Knoten, Granuloma anulare und Necrobiosis lipoidica in Betracht zu ziehen.

Angiosarkome sind einerseits sehr vielfältig in ihrer klinischen und histologischen Erscheinungsform und Ätiopathogenese [63], andererseits selten am Nagel. Von allen ist das Kaposi-Sarkom noch am häufigsten. Die Nagelregion wird meist sekundär in Mitleidenschaft gezogen. Klinisch handelt es sich um rote Flecken, die zu blauroten Plaques und schließlich Tumoren werden, die meist recht ausgeprägte Hämorrhagien mit Hämosideri-

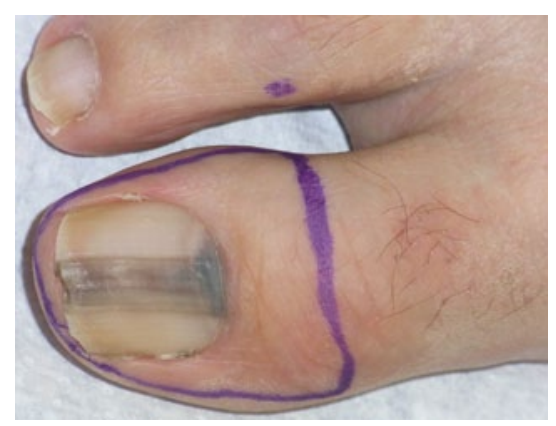

Abb. $5 \Delta$ Von der Nagelmatrix ausgehendes Melanom, das im Lunulabereich dunkler und etwas breiter ist. Die Exzisionslinien sind eingezeichnet

nablagerungen aufweisen und dadurch einen braunen Farbton annehmen. Sie können auch als Cornu cutaneum imponieren [64]. Klinische Unterschiede zwischen dem endemischen, epidemischen und dem bei der erworbenen Immunschwäche auftretenden Kaposi-Sarkom bestehen nicht. Differenzialdiagnostisch ist das Pseudo-Kaposi-Sarkom bei akraler Hyperstomie und bei hyperplastischer Akroangiodermatitis abzugrenzen.

Von den anderen Angiosarkomtypen wurden nur Einzelfälle am Nagel beschrieben.

Chondrosarkome sind die häufigsten malignen Knochentumoren der Hand, sie sind aber sehr selten im Nagelapparat. Im Gegensatz zu den benignen Chondromen sind sie schmerzhaft. Röntgenologisch sieht man eine aufgehellte Knochenstruktur und oft Kalkspritzer. Prognostisch sind die apikalen Tumoren günstiger. Chondrosarkome kommen auch beim hereditären Syndrom multipler Exostosen [65], beim multiplen EnchondromSyndrom Ollier [66] und beim MaffucciKast-Syndrom [67] vor, die alle auch die Nagelregion betreffen können.

\section{Merkel-Zell-Karzinom}

Das Merkel-Zell-Karzinom ist ein seltener maligner Tumor, der vor 40 Jahren unter der Bezeichnung trabekuläres Karzinom der Haut beschrieben wurde [68]. Es ist bei Männern etwa doppelt so häufig wie bei Frauen. Die meisten Patienten sind hellhäufig, und lichtexponierte Haut ist daher vornehmlich betroffen, selten

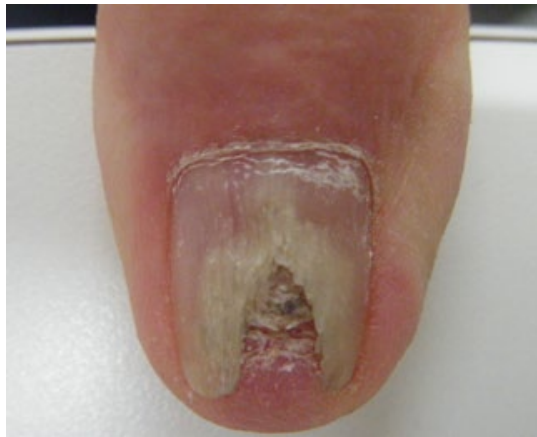

Abb. $6 \triangle$ Amelanotisches Nagelbettmelanom bei einem 68-jährigen Mann. Die Onycholyse war jahrelang als traumatisch durch häufige Gartenarbeit bedingt angesehen worden

auch die Nagelregion. Kürzlich wurde das Merkel-Zell-Polyomavirus (MCPyV) bei ca. $80 \%$ der Fälle identifiziert $[69,70]$. Die Tumoren sind halbkugelig erhaben, violett, schmerzhaft und schnell wachsend [71]. Granulationsgewebe kann imitiert werden [72]. Merkel-Zell-Karzinome haben eine hohe Rezidivneigung, und die großzügige Amputation ist daher bei Vorkommen an der Endphalanx indiziert. Differenzialdiagnostisch sind verschiedene Angiome und andere gefäßreiche Tumoren zu erwägen.

\section{Andere maligne neurogene Tumoren}

Das Ewing-Sarkom kommt skelettal und extraskelettal vor. Das Letztere ist zytogenetisch und molekulargenetisch identisch mit dem primären malignen peripheren neuroektodermalen Tumor (PNET). Sowohl das Ewing-Sarkom als auch der PNET kommen an Kopf, Stamm und Extremitäten vor. Die Lokalisation an der Fingerspitze und subungual ist sehr selten $[73,74,75]$. Ein Fall wurde subungual beobachtet (F. Facchetti, persönliche Mitteilung 2012). Die klinische Diagnose ist unspezifisch, und nur die Histologie mit Immunhistochemie kann sie korrekt stellen.

In Japan wurde ein Fall eines malignen metastasierenden Granularzelltumors am Nagel beobachtet [76].

\section{Maligne Systemerkrankungen}

Gelegentlich kommt es zu einer Beteiligung der Nagelregion im Rahmen von 


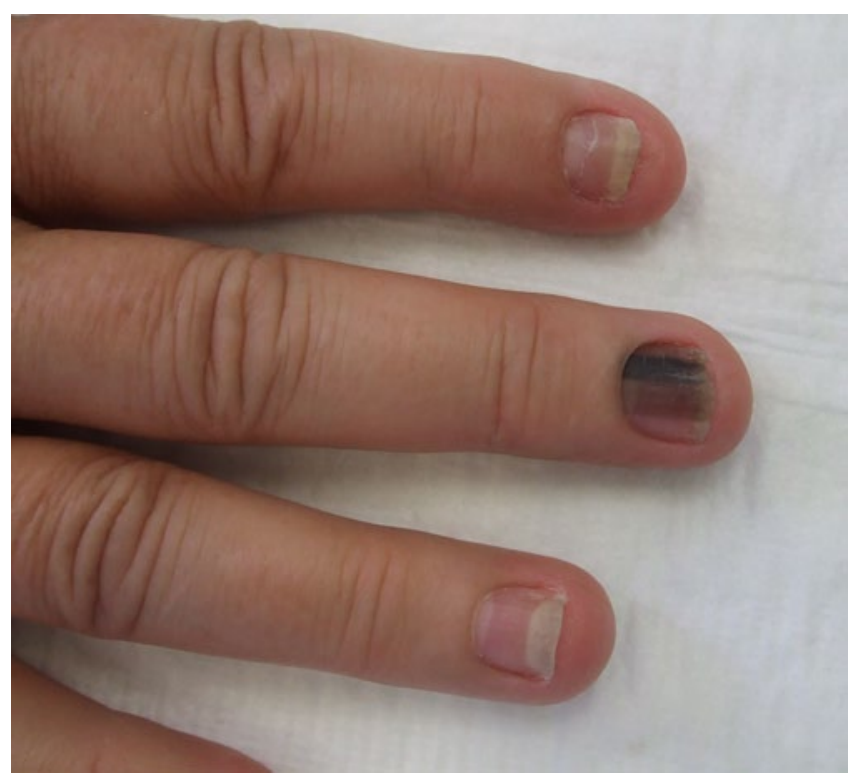

Abb. $7<$ Subunguales Melanoma in situ bei einer 63-jährigen Frau am rechten Mittelfinger. Die Melanonychie verläuft longitudinal nimmt ca. drei Viertel der Nagelbreite ein und ist in sich sehr unregelmäßig
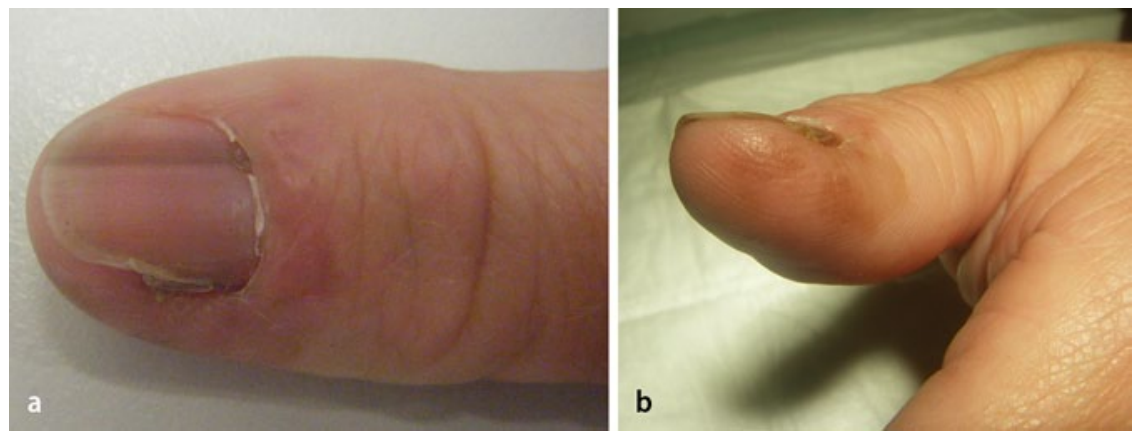

Abb. $8 \Delta$ Subunguales Melanom am Daumen einer 64-jährigen Frau mit Nageldystrophie und gut erkennbarem Hutchinson-Zeichen. a Dorsalansicht. b Seitenansicht mit ausgedehntem Hutchinson-Zeichen

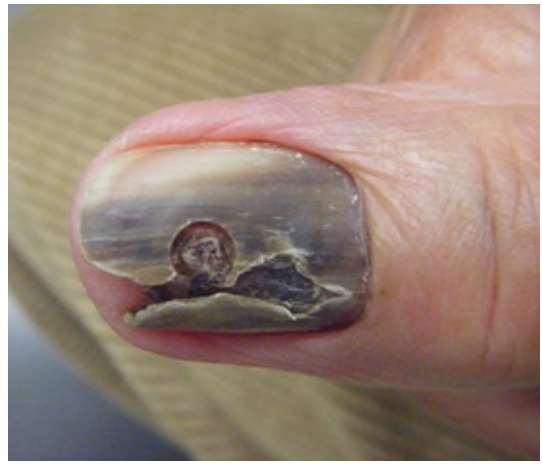

Abb. $9 \Delta$ Subunguales Melanom des Daumens bei einem 78-jährigen Mann. Der Nagel war schon vor der alio loco durchgeführten Stanzbiopsie dystrophisch

Leukämien und malignen Lymphomen. Während follikulotropische Lymphome gelegentlich vorkommen, ist eine Beteiligung der Nägel selten, und oft lässt sich dabei nur histologisch klären, ob es sich um eine direkte Nagelbeteiligung oder
Beim Plasmozytom mit Amyloidose kann es zu schleichender Nageldystrophie kommen, die sehr an einen atrophischen Lichen planus unguium erinnert.

Bei den Leukämien gibt es eine Reihe unspezifischer Nagelveränderungen wie Blässe des Nagelbetts, Nagelsplittern, parunguale Hämorrhagien und pernioartige Veränderungen an den Akren [79].

\section{Melanom}

Das Nagelmelanom ist zweifellos der wichtigste, weil mit der häufigste und maligneste Tumor.

\section{$>$ Etwa 1,5-2,5\% aller Melanome bei hellhäutigen Menschen sind unguale Melanome.}

Damit ist die Nagelregion im Vergleich zu ihrer Gesamtkörperoberfläche, die nur einen Bruchteil eines Prozentes beträgt, deutlich überrepräsentiert [80]. Die absolute Zahl von Nagelmelanomen ist bei Schwarzafrikanern und Asiaten vergleichbar mit der bei Hellhäutigen, der Prozentsatz ist mit 10 bis über $20 \%$ jedoch wesentlich höher, weil Melanome der übrigen Haut bei ihnen viel seltener sind. Obwohl sie auch bereits bei Kindern beobachtet werden (• Abb. 4, [81]), liegt der Erkrankungsgipfel bei 50 bis 60 Jahren. Etwa zwei Drittel bis drei Viertel der Nagelmelanome sind pigmentiert, wobei diese überwiegend von der Matrix ausgehen (• Abb.5), während Nagelbettmelanome meist amelanotisch sind (• Abb. 6). Insbesondere bei pigmentierten Tumoren könnte die Diagnose frühzeitig korrekt gestellt werden, jedoch zeigen $\mathrm{Pa}$ tientenkollektive von über 100 Nagelmelanomen mit einem mittleren vertikalen Durchmesser von $4 \mathrm{~mm}$ und dicker, dass das leider sehr häufig nicht der Fall ist, auch wenn ein brauner Längsstreifen jahrelang bestanden, sich allmählich verbreitert und sich sogar eine Nageldystrophie entwickelt hatte. Viele Melanome wurden erst anlässlich eines akuten Traumas der Finger- oder Zehenspitze diagnostiziert. Jahrelange Fehlbehandlung selbst als Onychomykose wurde mehrmals beschrieben [80].

An ein Melanom muss unbedingt gedacht werden, wenn sich eine Melanony- 

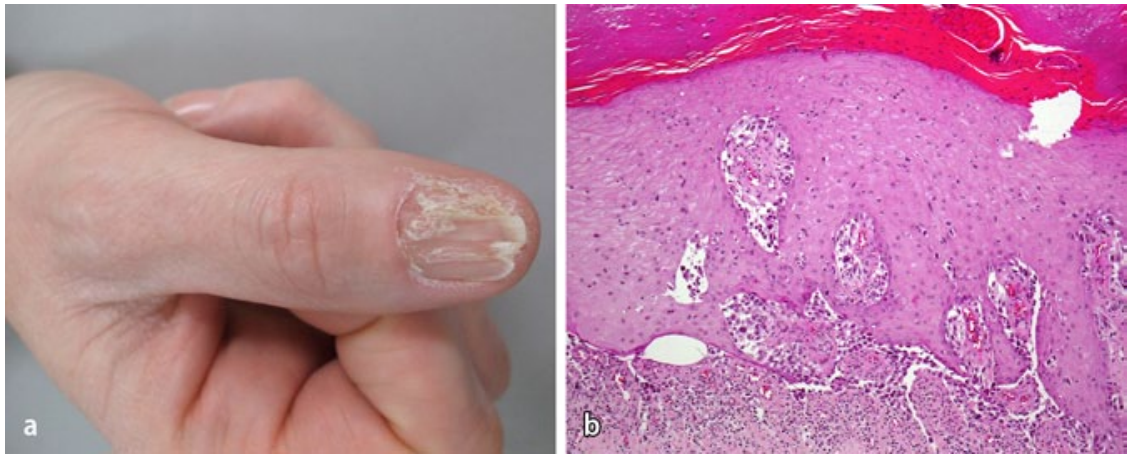

Abb. $10 \Delta$ Amelanotisches invasives Melanom am Daumen einer 42-jährigen Frau. a Klinischer Befund. b Die Histologie zeigt an der Grenze zur Epidermis und subepithelial relativ kleine Melanomzellen mit hyperchromatischen Kernen und ohne Pigmentierung

chie bei einem Erwachsenen entwickelt, wenn sie über $5 \mathrm{~mm}$ breit ist (• Abb. 7), wenn sie proximal breiter ist als distal, wenn sich eine periunguale Pigmentierung, das Hutchinson-Zeichen, entwickelt (• Abb. 8), wenn der Nagel dystrophisch wird (• Abb.9) und wenn ein blutender Tumor auftritt (• Abb. 10). Dermatoskopisch kann man ein Mikro-HutchinsonZeichen erkennen und gut von einem Pseudo-Hutchinson, der auf Durchscheinen des Pigmentes durch die Kutikula beruht, unterscheiden. Grundsätzlich muss bei jedem blutenden Tumor bei Erwachsenen, wenn nicht eindeutig ein Unguis incarnatus vorliegt, eine histologische Untersuchung des exzidierten Materials vorgenommen werden. Die Dermatoskopie kann gelegentlich die Diagnose eines subungualen Melanoms sicherer machen, wenn sie eine unregelmäßige Streifung erkennen lässt; sie ersetzt aber keinesfalls die Histologie [82].

Der diagnostische Goldstandard ist die histologische Untersuchung [80]. Dafür ist in den meisten Fällen eine oberflächliche Tangentialbiopsie ausreichend, die nicht nur die Unterscheidung von Melanin- und anderen Pigmentierungen sowie von gut- und bösartig erlaubt, sondern auch die Bestimmung der Eindringtiefe ermöglicht $[83,84]$. Ergibt die Histopathologie ein unguales Melanom, wird die komplette Exzision des Nagelorgans angeschlossen [80]. Die intraoperative direkte Matrixdermatoskopie ist der Dermatoskopie der Nagelplatte deutlich überlegen [85]. Jüngst wurde auch die intraoperative konfokale Laserscanningmikroskopie vorgestellt [86].
Die Behandlung des Nagelmelanoms richtet sich nach Eindringtiefe und Ausdehnung. Beim In-situ- und früh invasiven Melanom ist die großzügige Exzision des gesamten Nagelorgans mit einem Sicherheitsabstand von ca. $6 \mathrm{~mm}$ zu allen Seiten angezeigt [87]. Beim HutchinsonZeichen sollte ein Sicherheitsabstand von $10 \mathrm{~mm}$ eingehalten werden. Mikroskopisch kontrollierte Chirurgie mit histologischer Kontrolle der Schnittränder mithilfe eines Melanozytenmarkers, z. B. Melan-A, ist sinnvoll, weil sich einzelne Melanozyten oft nicht im HE-Schnitt darstellen. Beim fortgeschrittenen invasiven Melanom, das meist an Nageldystrophie und/ oder einem blutenden Tumor erkennbar ist [88], wird die Amputation empfohlen. Vergleichende Untersuchungen haben gezeigt, dass die Amputationshöhe - im Os metacarpale/metatarsale, metacarpo-/metatarsophalangeal, proximal oder distal interphalangeal - keinen Einfluss auf das Überleben hat, was auch für die lokale finger- bzw. zehenerhaltende Operation des frühen ungualen Melanoms spricht $[80,87]$.

Zur Durchführung einer Exstirpation des Sentinellymphknotens gibt es keine allgemeinen Richtlinien. Sie kann aber frühzeitig Metastasen aufdecken [89].

\section{Metastasen}

Am Nagelorgan wurde eine Reihe verschiedener Metastasen beschrieben. Meistens handelt es sich um Knochenmetastasen der distalen Phalanx, die zu einem Trommelschlegelfinger führen. Eine reine Nageldestruktion ohne Knochenbeteiligung ist selten $[90,91]$.

\section{Fazit für die Praxis}

- Das Nagelorgan ist eine komplizierte Struktur, die mit allen anderen Bestandteilen der Finger- bzw. Zehenspitze eine funktionelle und anatomische Einheit bildet.

- Entsprechend der enormen Anzahl verschiedener Zellen und Gewebe gibt es eine sehr große Vielfalt an Tumoren, zu denen noch die Nagelbeteiligung im Rahmen maligner Systemkrankheiten und Metastasen hinzukommt.

- Abgesehen von wenigen Nagelmalignomen sind die meisten klinisch unspezifisch.

- Ein hohes Maß an Aufmerksamkeit und Verdacht ist erforderlich, um maligne Neoplasmen des Nagels frühzeitig zu erkennen und einer Therapie zuführen zu können.

\section{Korrespondenzadresse}

\section{Prof. Dr. E. Haneke}

Dermaticum Freiburg

Schlippehof 5, 79110 Freiburg

haneke@gmx.net

\section{Einhaltung ethischer Richtlinien}

Interessenkonflikt. E. Haneke gibt an, dass kein Interessenkonflikt besteht.

Dieser Beitrag beinhaltet keine Studien an Menschen oder Tieren.

\section{Literatur}

1. Lecerf P, Richert B, Theunis A, André J (2013) A retrospective study of squamous cell carcinoma of the nail unit diagnosed in a Belgian general hospital over a 15-year period. J Am Acad Dermatol 69:253-261

2. Haneke E, Bragadini LA, Mainusch O (1997) Enfermedad de Bowen de células claras del aparato ungular. Act Terap Dermatol 20:311-313

3. Haneke E (1991) Epidermoid carcinoma (Bowen's disease) of the nail simulating acquired ungual fibrokeratoma. Skin Cancer 6:217-221

4. Baran R, Simon C (1988) Longitudinal melanonychia: a symptom of Bowen's disease. J Am Acad Dermatol 18:1359-1360

5. Sass U, André J, Stene JJ, Noel JC (1998) Longitudinal melanonychia revealing an intraepidermal carcinoma of the nail apparatus: detection of integrated HPV-16 DNA. J Am Acad Dermatol 39:490-493 
6. Lambiase MC, Gardner TL, Altman CE, Albertini JG (2003) Bowen disease of the nail bed presenting as longitudinal melanonychia: detection of human papillomavirus type 56 DNA. Cutis 72:305-309 (quiz 296)

7. Stetsenko GY, McFarlane RJ, Chien AJ et al (2008) Subungual Bowen disease in a patient with epidermodysplasia verruciformis presenting clinically as longitudinal melanonychia. Am J Dermatopathol 30:582-585

8. Saito T, Uchi H, Moroi Y et al (2012) Subungual Bowen disease revealed by longitudinal melanonychia. J Am Acad Dermatol 67:e240-e241

9. Park SW, Lee DY, Mun GH (2013) Longitudinal melanonychia on the lateral side of the nail: a sign of Bowen disease associated with human papillomavirus. Ann Dermatol 25:378-379

10. Lipoff JB, Scope A, Busam KJ, Nehal KS (2008) Melanonychia following Mohs surgery for recurrent squamous cell carcinoma in situ of the nail bed. J Cutan Med Surg 12:194-197

11. Mitsuishi T, Tetsutaro S, Toshihiko M et al (1997) The presence of mucosal human papillomavirus in Bowen's disease of the hands. Cancer 79: 1911-1917

12. Sau P, McMarlin S, Sperling L et al (1994) Bowen's disease of the nail bed and periungual area. Arch Dermatol 130:204-209

13. Kreuter A, Gambichler T, Pfister H, Wieland U (2009) Diversity of human papil- lomavirus types in periungual squa- mous cell carcinoma. Br J Dermatol 161:1262-1269

14. Turowski CB, Ross AS, Cusack CA (2009) Human papillomavirus-associated squamous cell carcinoma of the nail bed in African-American patients. Int J Dermatol 48:117-120

15. Zabawski EJ Jr, Washak RV, Cohen JB et al (2001) Squamous cell carcinoma of the nail bed: is finger predominance another clue to etiology? A report of 5 cases. Cutis 67:59-64

16. Grundmeier N, Hamm H, Weissbrich B et al (2011) High-risk human papillomavirus infection in Bowen's disease of the nail unit: report of three cases and review of the literature. Dermatology 223:293-300

17. Nordin P, Steinquist B, Hansson B-G (1994) Joint occurrence of human papllomavirus type 16 DNA in Bowen's disease on a finger and in dysplasia of the vulva and the uterine cervix. Br J Dermatol 131:740

18. Forslund O, Nordin P, Andersson K et al (1997) DNA analysis indicates patient-specific human papillomavirus type 16 strains in Bowen's disease on fingers and in archival samples from genital dysplasia. Br J Dermatol 136:678-682

19. Eliezri YD, Silverstein SJ, Nuovo GJ (1990) Occurrence of human papillomavirus type 16DNA in cutaneous squamous and basal cell neoplasms. J Am Acad Dermatol 23:836-842

20. Seçilmiş Kerimoğlu O, Doğan NU, Tazegül A et al (2012) Lichen simplex chronicus that accompanies anogenital warts during the childhood. Case Report Med 2012:192767

21. Figus $A$, Kanitkar S, Elliot D (2006) Squamous cell carcinoma of the lateral nail fold. J Hand Surg $\mathrm{Br}$ 31:216-220

22. Mikhail G (1984) Subungual epidermoid carcinoma. J Am Acad Dermatol 11:291-298

23. Arumugan M (2007) Squamous cell carcinoma of the thumb nail bed. Ind J Dermatol Venereol Leprol 73:445 (Netcase)

24. Sbai MA, Balti W, Boussen M et al (2009) Lateral nail squamous cell carcinoma: case report. Tunis Med 87:86-88
25. Goldminz D, Bennett RG (1992) Mohs micrographic surgery of the nail unit. J Dermatol Surg Oncol 18:72-725

26. Modi G, Jacobs AA, Orengo IF et al (2009) Combination therapy with imiquimod, 5 -fluorouracil, and tazarotene in the treatment of extensive radiationinduced Bowen's disease of the hands. Dermatol Chir 35:1-7

27. Weisenseel P, Prinz J, Korting H (2006) Behandlung eines parungualen HPV73-positiven Morbus Bowen mit Imiquimodcreme. Hautarzt 57:309-310, 312

28. Yaparpalvi R, Mahadeia PS, Gorla GR, Beitler JJ (2003) Radiation therapy fort he salvage of unresectable subungual squamous cell carcinoma. Dermatol Surg 29:294-296

29. Sachse MM, Schmoll J, Wagner G (2011) Cornu cutaneum-like HPV 45 positive subungual squamous cell carcinoma. J Dtsch Dermatol Ges 9:226-228

30. Harwood M, Telang GH, Robinson-Bostom L, Jellinek N (2008) Melanoma and squamous cell carcinoma on different nails of the same hand. J Am Acad Dermatol 58:323-326

31. Wilkins K, Turner R, Dolev JC et al (2006) Cutaneous malignancy and human immunodeficiency virus disease. J Am Acad Dermatol 54:189-206

32. Baran R, Haneke E (1990) Epithelioma cuniculatum of the terminal phalanx - clinical aspects. XI Int Cong Dermatol Surg. Florenz, 12.-15.10.1990. Book of Abstracts 53

33. Haneke E, Baran R (1990) Epithelioma cuniculatum. Histological and immunohistochemical aspects. XI Int Cong Dermatol Surg. Florenz, 12.15.10.1990, Book of Abstracts 55

34. Kowalzick L, Blum R, Iliev D, Seidel R (2003) HPV16 positives subunguales „warty squamous cell carcinoma" (warziges Karzinom) der Hand. Akt Dermatol 29:473-476

35. Kurashige Y, Kato Y, Hobo A, Tsuboi R (2013) Subungual verrucous carcinoma with bone invasion. Int J Dermatol 52:217-219

36. Sellheyer K, Krahl D (2008) Basal cell (trichoblastic) carcinoma. Common expression pattern for epithelial cell adhesion molecule links basal cell carcinoma to early follicular embryogenesis, secondary hair germ, and outer root sheath of the vellus hair follicle: a clue to the adnexal nature of basal cell carcinoma? J Am Acad Dermatol 58:158-167

37. Eisenklam D (1931) Über subunguale Tumoren. Wien Klin Wochenschr 44:1192-1193

38. Rudolph RI (1987) Subungual basal cell carcinoma presenting as longitudinal melanonychia. J Am Acad Dermatol 16:229-233

39. Herzinger T, Flaig M, Diederich R, Röcken M (2003) Basal cell carcinoma of the toenail unit. J Am Acad Dermatol 48:277-278

40. Forman SB, Ferringer TC, Garrett AB (2007) Basal cell carcinoma of the nail unit. J Am Acad Dermatol 56:811-814

41. Kao GF, Helwig EB, Graham JH (1987) Aggressive digital papillary adenoma and adenocarcinoma. A clinicopathological study of 57 patients with histochemical, immunopathological and ultrastructural observations. J Cutan Pathol 14:129-146

42. Duke WH, Sherod TT, Lupton GP (2000) Aggressive digital papillary carcinoma (aggressive digital papillary adenoma and adenocarcinoma revisited). Am J Surg Pathol 24:775-784

43. Matysik TS, Port M, Black JR (1990) Aggressive digital papillary adenoma: a case report. Cutis 46 : 125-127
44. Bazil MK, Henshaw RM, Werner A, Lowe EJ (2006) Aggressive digital papillary adenocarcinoma in a 15-year-old female. J Pediatr Hematol Oncol 28:529-530

45. Frey J, Shimek C, Woodmansee C et al (2009) Aggressive digital papillary adenocarcinoma: a report of two diseases and review of the literature. J Am Acad Dermatol 60:331-339

46. Singla AK, Shearin JC (1997) Aggressive surgical treatment of digital papillary adenocarcinoma. Plast Reconstr Surg 99:2058-2060

47. Pinkus H, Mehregan AH (1963) Epidermotropic eccrine carcinoma. Arch Dermatol 88:597-606

48. Mehregan AH, Hashimoto K, Rahbari H (1983) Eccrine adenocarcinoma. A clinicopathologic study of 35 cases. Arch Dermatol 119:104-114

49. Van Gorp J, Putte SC van der (1993) Periungual eccrine porocarcinoma. Dermatology 187:67-70

50. Requena L, Sanchez M, Aguilar P et al (1990) Periungual porocarcinoma. Dermatologica 180: 177-180

51. Moussallem CD, Abi Hatem NE, El-Khouri ZN (2008) Malignant porocarcinoma of the nail fold: a tricky diagnosis. Dermatol Online J 14:8

52. Zina AM, Bundino S, Pippione MG (1982) Pigmented hidroacanthoma simplex with porocarcinoma. Light and electron microscopic study of a case. J Cutan Pathol 9:104-112

53. Geraci TL, Janis L, Jenkinson S, Stewart R (1987) Mucinous (adenocystic) sweat gland carcinoma of the great toe. J Foot Surg 26:520-523

54. Argenyi ZB, Nguyen AV, Balogh K et al (1992) Malignant eccrine spiradernoma. A clinicopathologic study. Am J Dermatopathol 14:381-390

55. Engel CJ, Meads GE, Joeph NG, Stavraky W (1991) Eccrine spiradenoma: a report of malignant transformation. Can J Surg 34:477-480

56. Nash J, Chaffins M, Krull E (2001) Hidradenocarcinoma. 59th Ann Meet Am Acad Dermatol, Washington, DC, 2-7 March 2001

57. Kasdan ML, Stutts JT, Kassan MA, Clanton JN (1991) Sebaceous gland carcinoma of the finger. J Hand Surg 16A:870-872

58. Coles M, Smith M, Rankin EA (1989) An unusual case of dermatofibrosarcoma protuberan. J Hand Surg 14A:135-138

59. Hashiro M, Fujio Y, Shoda Y, Okumura M (1995) A case of dermatofibrosarcoma protuberans on the right first toe. Cutis 56:281-282

60. Dumas V, Euvrard S, Ligeron C et al (1998) Dermatofibrosarcome de Darier - Ferrand sous-unguéal. Ann Dermatol Vénéréol 125(Suppl 3):S93

61. Chase DR, Enzinger FM (1985) Epithelioid sarcoma. Am J Surg Pathol 9:241-263

62. Carloz B, Bioulac P, Gavard J et al (1991) Recidives multiples d'un sarcome épithélioïde. Ann Dermatol Vénéréol 118:623-628

63. Requena L, Sangueza OP (1998) Cutaneous vascular proliferations. Part III. Malignant neoplasms, other cutaneous neoplasms with significant vascular component, and disorders erroneously considered as vascular neoplasms. J Am Acad Dermatol 38:143-175 (quiz 176-178)

64. Oludiran OO, Ekanem VJ (2011) Cutaneous horns in an African population. J Cutan Aesthet Surg 4:197-200

65. Solomon $L$ (1974) Chondrosarcoma in hereditary multiple exostosis. S Afr Med J 48:671-676

66. Nakajima H, Ushigome S, Fukuda J (1988) Case report 482: chondrosarcoma (grade 1) arising from the right second toe in patient with multiple enchondroma. Skeletal Radiol 17:289-292 


\section{Leitthema}

67. Cremer H, Gullotta F, Wolf L (1981) The MafucciKast syndrome. Dychondroplasia with hemangiomas and frontal lobe astrocytoma. J Cancer Res Clin Oncol 101:231-237

68. Toker C (1972) Trabecular carcinoma of the skin. Arch Dermatol 105:107-110

69. Feng H, Shuda M, Chang Y, Moore PS (2008) Clonal integration of a polyomavirus in human Merkel cell carcinoma. Science 319(5866):1096-1100

70. Helmbold P, Lahtz C, Enk A et al (2009) Frequent occurrence of RASSF1A promoter hypermethylation and Merkel cell polyomavirus in Merkel cell carcinoma. Mol Carcinog 48:903-909

71. Engelmann L, Kunze J, Haneke E (1991) Giant neuroendocrine carcinoma of the skin (Merkel cell tumour). Skin Cancer 6:211-216

72. Goldenhersh MA, Prus D, Ron N, Rosenmann E (1992) Merkel cell tumor masquerading as granulation tissue on a teenager's toe. Am J Dermatopathol 14:560-563

73. Dick HM, Francis KC, Johnston AD (1971) Ewing's sarcoma of the hand. J Bone Joint Surg 53A: 345-348

74. Steens SC, Kroon HM, Taminiau AH et al (2007) Nail-patella syndrome associated with Ewing sarcoma. JBR-BTR 90:214-215

75. Lee CS, Southey MC, Slater H et al (1995) Primary cutaneous Ewing's sarcoma/peripheral primitive neuroectodermal tumors in childhood. A molecular, cytogenetic, and immunohistochemical study. Diagn Mol Pathol 4:174-181
76. Urabe A, Imayama S, Yasumoto S et al (1991) Malignant granular cell tumor. J Dermatol 18: 161-166

77. Harland E, Dalle S, Balme B et al (2006) Ungueotropic T-cell lymphoma. Arch Dermatol 142: 1071-1073

78. Parmentier L, Dürr C, Vassella E et al (2010) Nail alterations in cutaneous $\mathrm{T}$ cell lymphoma: successful treatment of specific nail infiltration with topical mechlorethamine only. Arch Dermatol 146 : 1287-1291

79. Hirschfeld H (1925) Leukämie und verwandte Zustände. In: Schittenhelm A (Hrsg) Handbuch der blutbildenden Organe, Bd 1. Springer, Berlin

80. Haneke E (2012) Ungual melanoma - controversies in diagnosis and treatment. Dermatol Ther 25:510-524

81. Tosti A, Piraccini BM, Cagalli A, Haneke E (2012) In situ melanoma of the nail unit in children. Report of two cases in fair-skinned caucasian children. Pediatr Dermatol 29:79-83

82. Lee MD, Humphreys T (2012) Nail biopsy for melanonychia. Skinmed 10:309-331

83. Haneke E (2011) Advanced nail surgery. J Cutan Aesthet Surg 4:167-175

84. Di Chiacchio N, Loureiro WR, Michalany NS, Haneke E (2013) La extirpación tangencial para el diagnóstico y tratamiento de la melanoniquia longitudinal. Tangential excision for the diagnosis and/ or treatment of longitudinal melanonychias (Engl \& Span). Dermatol Rev Mex 57:289-293
85. Di Chiacchio ND, Farias DC de, Piraccini BM et al (2013) Consensus on melanonychia nail plate dermoscopy. An Bras Dermatol 88:309-313

86. Debarbieux S, Hospod V, Depaepe L et al (2012) Perioperative confocal microscopy of the nail matrix in the management of in situ or minimally invasive subungual melanomas. Br J Dermatol 167:828-836

87. Haneke E, Binder D (1978) Subunguales Melanom mit streifiger Nagelpigmentierung. Hautarzt 29:389-391

88. Haneke E (1986) Pathogenese der Nageldystrophie beim subungualen Melanom. Verh Dtsch Ges Pathol 70:484

89. Haenssle HA, Buhl T, Holzkamp R et al (2012) Squamomelanocytic tumor of the nail unit metastasizing to a sentinel lymph node: a dermoscopic and histologic investigation. Dermatology 225:127130

90. Baran R, Tosti A (1994) Metastatic carcinoma to the terminal phalanx of the big toe: report of two cases and review of the literature. J Am Acad Dermatol 31:259-263

91. Chang P, Rosales D, Calderón G, García Aceituno LF (2013) Metástasis de un adenocarcinoma ductal invasivo al aparato ungueal. Dermatología CMQ 11:257-260

\section{Hier steht eine Anzeige.}

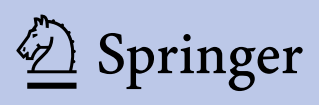

